\title{
Abundancia, distribución espacial y temporal de aves playeras (Orden: Charadriiformes) en Marismas Nacionales, México
}

\author{
Luis F. Mendoza ${ }^{1}$, Roberto Carmona ${ }^{1,2}$, Gustavo de la Cruz-Agüero ${ }^{3}$, Pablo del Monte-Luna ${ }^{3}$ \\ \& José de la Cruz-Agüero ${ }^{3}$ \\ 1. Departamento Académico de Ciencias Marinas y Costeras, Universidad Autónoma de Baja California Sur, La Paz, \\ Baja California Sur 23080, México; luis_kiwi@hotmail.com, beauty@uabcs.mx \\ 2. Pronatura Noroeste A.C. Calle Décima No 60, Ensenada, Baja California 22800, México. \\ 3. Centro Interdisciplinario de Ciencias Marinas (CICIMAR-IPN). P.O. Box 592. La Paz, Baja California Sur 23096, \\ México; gaguero@ipn.mx,pdelmontel@ipn.mx, jcruz@ipn.mx
}

Recibido 26-XI-2018. Corregido 30-VII-2019. Aceptado 09-VIII-2019.

\begin{abstract}
Abundance, spatial, and temporal distribution of shorebirds (Order: Charadriiformes) at Marismas Nacionales, Mexico. Northwest Mexico is an important region for shorebirds associated with an extensive series of wetlands. However, for many of these wetlands, basic information about shorebirds communities like abundance, population trends, and richness are limited. Currently, wintering and stopover sites are critical for conservation because many populations of shorebirds have declined in the last decades. Marismas Nacionales $(\mathrm{MN})$ is an important tropical wetland with a dynamic ecosystem and where shorebirds studies are limited to few wintering aerial and ground surveys. Our goals were analyzing shorebirds abundance and spatial and temporal distribution patterns in 2010-2011 season. We selected eight monitoring sites from two prospective visits to the study area. We observed shorebirds monthly between November 2010 and June 2011 to analyze richness and abundance patterns by site and month. Additionally, we describe specific spatial and temporal distribution for dominant species. A total of 27 shorebirds species and one genus, with a minimum global abundance of 136236 individuals were found. Shorebird abundance at $\mathrm{MN}$ is among the most important in the region with around $10 \%$ of total abundance in northwest Mexico; therefore, $\mathrm{MN}$ is a priority conservation site for this group of birds. Additionally, MN presents a suitable habitat for breeding of some shorebirds species such as Snowy and Wilson' Plover, Killdeer, Northern Jacana, Black-necked Stilt and Pacific American Oystercatcher. Dominant species were: Western Sandpiper (33.5\% of total in MN), American Avocet (31\%) and Dowitchers (17\%). These taxa are very common in others wetlands in Northwest Mexico region. Spatially, shorebirds were distributed in three sites: Chumbeño lagoon (37\% of abundance total), Las Garzas-Chihuin lagoons (24.2 \%) and La Polca lagoon (24\%). The less used sites by shorebirds are located in the southern part of MN. Our results update MN information and can help to establish monitoring programs in the area.
\end{abstract}

Key words: tropical lagoon; shorebirds; non-breeding season; Nayarit; avian community.

Mendoza, L. F., Carmona, R., de la Cruz-Agüero, G., del Monte-Luna, P. \& de la CruzAgüero, J. (2019). Abundancia, distribución espacial y temporal de aves playeras (Orden: Charadriiformes) en Marismas Nacionales, México. Revista de Biología Tropical, 67(4), 1077-1088.

Las aves playeras (Orden Charadriiformes) están entre las aves acuáticas más abundantes y distribuidas en las costas del Pacífico Americano. En la región neártica se localizan las áreas de reproducción de 49 especies de playeros, mismas que pasan su temporada no reproductiva en áreas que van desde el sur de Canadá hasta la Patagonia en Argentina (Myers, Morrison, Antas, Harrington, Lovejoy, Sallaberry, Senner, \& Tarak, 1987; Hayman, Marchant, 
\& Prater, 1988; O’Brien, Crossley, \& Karlson, 2006). Las estrategias migratorias de las aves playeras están entre las más complejas debido a los diferentes patrones de distribución y uso de los humedales, lo que les permite aprovechar recursos alimenticios cíclicos en lugares que no soportarían una explotación continua (Myers et al., 1987; Howes \& Bakewell, 1989; Skagen \& Knopf, 1994).

La distribución espacial de las aves playeras dentro de un humedal es un proceso dinámico y está influenciado por varios factores, tanto bióticos (e.g. abundancia y disponibilidad de alimento, depredadores) como abióticos (e.g. tipo de sustrato, compactación del sedimento, nivel de mareas). Estos factores llegan a explicar la mayoría de las variaciones en las distribuciones y abundancias de las especies (Yates et al., 1993; Danufsky \& Colwell, 2003).

En México, la región noroeste concentra las mayores abundancias de aves playeras durante la época no reproductiva, debido a la presencia de extensos humedales muchos de los cuales están incluidos en la Red Hemisférica de Reservas para Aves Playeras (RHRAP) y forman parte del corredor migratorio del Pacífico (Harrington, 1993; Page, Palacios, Alfaro, González, Stenzel, \& Jungers, 1997; Engilis, Oring, Carrera, Nelson, \& Martínez-López, 1998; Morrison \& Ross, 2009). Marismas Nacionales (MN) es uno de los humedales más extensos de esta región y su complejo ecosistema está conformado por diversos ambientes (Harrington, 1993; Morrison, Ross, \& Guzmán, 1994). MN tiene varios reconocimientos nacionales e internacionales por su valor para las aves acuáticas. En 1992 el área fue reconocida como sitio clave para la conservación de aves playeras por la RHRAP, en su categoría de Importancia Internacional por ser utilizada por más de 100000 individuos al año. En 1995 fue designada como Sitio Ramsar para la conservación de humedales y desde 1998 es considerada un Área de Importancia para la Conservación de las Aves (AICA, Cervantes-Abrego, 2000).

Actualmente en $\mathrm{MN}$ se ha generado poca información sobre la abundancia y distribución de las aves playeras, sobre todo en el aspecto temporal. Los trabajos descriptivos sobre la abundancia de este grupo comenzaron hace más de 20 años con censos aéreos invernales. Por ejemplo, Harrington (1993) recorrió en una sola ocasión la parte norte de Marismas Nacionales (Sinaloa), con base en la cobertura censada estimó, por extrapolación, cerca de 101000 individuos. Posteriormente, también mediante un conteo aéreo, Morrison et al. (1994) recorrieron la totalidad de MN y registraron aproximadamente 104000 individuos de aves playeras.

Después de estos censos aéreos no existe información disponible sobre las aves playeras en MN. En 2011 se presentó un trabajo sobre la distribución espacio-temporal de aves playeras en la porción nayarita de MN (Ortega-Solis, 2011). Este estudio no cubrió la totalidad del área y el esfuerzo de observación varió por sitio y por fecha, no obstante, se estimó una abundancia aproximada de 64000 aves y ofrece una descripción adecuada de los sitios de mayor importancia numérica, al menos para la parte central de MN.

La información escasa sobre aves playeras en $\mathrm{MN}$ ha dificultado entender la dinámica de este grupo de aves en la zona, además los censos a la fecha no han considerado todos los sitios potencialmente importantes. Esta información es fundamental para desarrollar estrategias de monitoreo y planes de conservación, sobre todo teniendo en cuanta que en las últimas décadas muchas poblaciones de aves playeras han disminuido debido a la pérdida o degradación del hábitat. Por lo anterior, el estudio presente tuvo como finalidad determinar la abundancia y distribución temporal y espacial de las aves playeras en MN durante la temporada no reproductiva.

\section{MATERIALES Y MÉTODOS}

Área de estudio: MN es un extenso humedal que se localiza en la costa del Pacífico mexicano, entre los Estados de Nayarit y Sinaloa $\left(21^{\circ} 32^{\prime} \mathrm{N}-105^{\circ} 15^{\prime} \mathrm{W} \& 22^{\circ} 45^{\prime}\right.$ $\left.\mathrm{N}-105^{\circ} 50^{\prime} \mathrm{W}\right)$. Tiene una superficie aproximada de 350000 ha (Bojórquez-Tapia, 
Díaz-Mondragón, \& Ezcurra, 2001) de las cuales 133000 ha forman parte de la Reserva de la Biosfera Marismas Nacionales Nayarit decretada en 2010 (DOF, 2010a; Fig. 1). MN presenta diversos hábitats como deltas, manglares, barras de arena, marismas, lagunas costeras y planicies lodosas (CONANP, 2005). El clima de la región es cálido subhúmedo y la precipitación promedio anual es de $1200 \mathrm{~mm}$ mismas que empiezan en mayo y se concentran de junio a octubre (CONAGUA, 2004; Berlanga-Robles, García-Campos, López-Blanco, \& Ruiz-Luna, 2010; Blanco et al., 2011). Las mareas dentro de $\mathrm{MN}$ son poco notorias y no se tiene un buen conocimiento sobre sus variaciones dentro de las lagunas costeras interiores. Por ejemplo, para Laguna Las Garzas-Chahuin (Nayarit), se indicó un intervalo de marea de apenas $3 \mathrm{~cm}$ verticales en noviembre de 2013, dicho intervalo coincidió incluso con el período de mareas vivas (Arce, Carmona, Miramontes, Ayala-Perez, Hernández-Alvarez, \& Mendoza, 2015).

La complejidad de los diferentes ambientes de MN hace difícil recorrerlos en su totalidad. Por lo anterior, se realizaron dos visitas

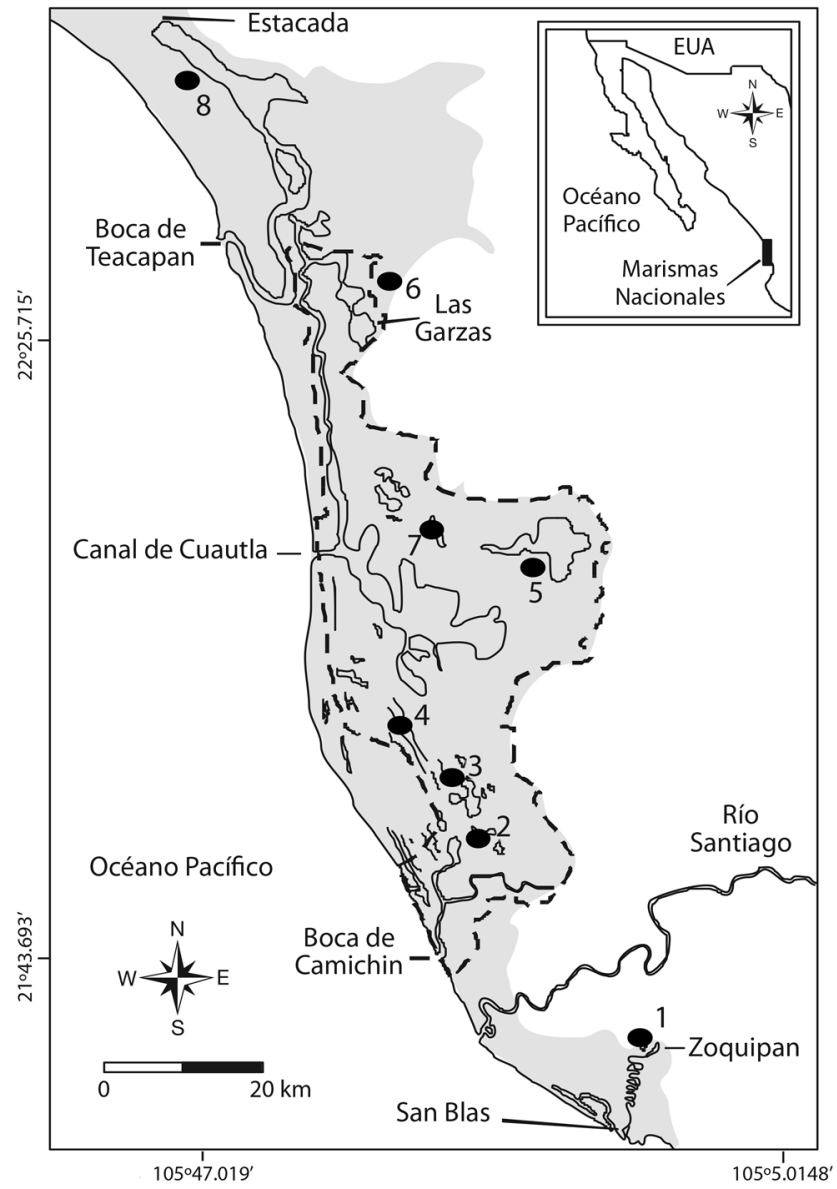

Fig. 1. Marismas Nacionales (Nayarit-Sinaloa). La zona sombreada representa el área del sistema y la línea discontinua muestra la parte decretada como Reserva de la Biosfera. Se indican las unidades de muestreo: 1= Zoquipan, 2= Batanga, $3=$ Mexcaltitán, 4= Cañadas, 5= Chumbeño, 6= Las Garzas-Chahuin, 7= Zoyata y $8=$ La Polca.

Fig. 1. Marismas Nacionales. Shade zone delimits the wetland area and discontinued line represent the area declared as Biosphere Reserve. Dark circles represent monitoring units: 1= Zoquipan, 2= Batanga, 3= Mexcaltitán, 4= Cañadas, 5= Chumbeño, 6= Las Garzas-Chahuin, 7= Zoyata y 8= La Polca. 
prospectivas en septiembre y octubre de 2010 . Se estima que se visitó aproximadamente el 85 $\%$ del área de $\mathrm{MN}$. Se recorrieron 79 sitios y se descartaron las zonas menos adecuadas para las aves playeras (e.g. cuerpos de agua profundos, vegetación densa, predominio de sustratos arenosos y rocosos). De esta forma, las unidades de muestreo elegidas se conformaron por ocho sitios: 1) Zoquipan, 2) La Batanga, 3) Laguna Grande de Mexcaltitán, 4) Las Cañadas, 5) Laguna El Chumbeño, 6) Lagunas Las GarzasChahuin, 7) Laguna Zoyata y 8) Laguna La Polca (Fig. 1).

Metodología: Los ocho sitios seleccionados se visitaron mensualmente para censar las aves playeras presentes entre noviembre 2010 y junio 2011. Este periodo permitió obtener información de la temporada no reproductiva y parte de la temporada reproductiva. Estudios sobre aves playeras neárticas de más de un año de duración en otros humedales del noroeste de México, han registrado bajas abundancias de este grupo de aves después de junio, debido a que la mayoría de estas se encuentran en sus áreas de reproducción en el norte del continente americano (Myers et al., 1987; Carmona, Arce, Ayala-Pérez, \& Danemann, 2011; Mendoza \& Carmona, 2013), es por ello que el estudio presente descarta estos meses (julio-agosto) por razones de logística.

Los censos fueron de tres tipos: (1) navegando por canales y lagunas en embarcaciones menores, (2) en automóvil sobre los caminos existentes que dan acceso a las áreas donde se concentran las aves playeras y (3) caminando por las áreas donde existen amplias zonas de inundación. Los censos mantuvieron el mismo protocolo de conteo en todos los meses y en las mismas áreas. Los conteos de las aves playeras se realizaron durante las mareas muertas y bajas, para minimizar el efecto de los movimientos locales de las aves, aunque el efecto por marea es limitado (ver área de estudio). Los conteos se realizaron con binoculares (10x) y telescopio (20-60x), cuando el tamaño de la parvada era superior a las 300 aves se realizaron estimaciones de las abundancias mediante el método de bloques, sugerido por Howes \& Bakewell (1989).

Se formaron dos equipos de trabajo, uno de ellos recorrió el sistema desde el norte (Laguna La Polca) y el otro desde el sur (Laguna Zoquipan), para encontrarse en Laguna las Garzas-Chahuin. Cada equipo de trabajo estuvo conformado por cuatro personas, dos observadores con experiencia y dos anotadores. Las observaciones iniciaron a las 07:00 h y finalizaron a las 17:00 h, cada visita tuvo una duración de cinco días de trabajo de campo.

Los análisis realizados incluyeron la descripción de las abundancias y riquezas mensuales de la comunidad de aves playeras. Los sitios se agruparon formando secciones para determinar la relevancia de estos con base a las abundancia y tendencias espaciales de las aves playeras. Para esto se formó un dendrograma utilizando el índice de similitud cuantitativa de Bray-Curtis y la técnica de Unión Media no Ponderada (Crisci \& López, 1983). La estimación general de la abundancia de aves playeras que utilizaron Marismas Nacionales se determinó sumando la abundancia máxima registrada por cada especie, independientemente del mes de observación (Tabla 1). De esta forma se minimiza la posibilidad de reconteo de las aves. Este es un criterio conservador propuesto por diferentes instancias internacionales, incluida la Red Hemisférica de Reservas para Aves Playeras, con la finalidad de reducir la sobreestimación de la abundancia de las aves playeras en un humedal determinado.

Se describieron también las tendencias en la distribución espacial y temporal de las especies de aves playeras con mayor abundancia. Dado que los criterios de clasificación son cualitativos (meses y sitios), para las especies dominantes se realizó una prueba de independencia $X^{2}$ múltiple donde se probó si la abundancia por especie fue independiente del sitio y mes de observación $(\alpha=0.05$ para todos los casos; Zar, 2010). Además, para estas especies se determinó el porcentaje poblacional registrado en Marismas Nacionales, utilizando los estimados más recientes (Andres et al., 2012). Particularmente para el Chorlo nevado 
TABLA 1

Aves playeras observadas durante la temporada 2010-2011 en Marismas Nacionales

TABLE 1

Shorebirds observed at Marismas Nacionales in 2010-2011 season

\begin{tabular}{|c|c|c|c|c|}
\hline Especies & Abundancia acumulada & $\begin{array}{c}\text { Máxima } \\
\text { estimación mensual }\end{array}$ & $\begin{array}{l}\text { Mes de máx. } \\
\text { estimación }\end{array}$ & Presencia \\
\hline Pluvialis squatarola & 1941 & 626 & Febrero & M \\
\hline Charadrius collaris & 18 & 12 & Enero & $\mathrm{R}$ \\
\hline Charadrius nivosus $* A$ & 1228 & 268 & Febrero & RI \\
\hline Charadrius wilsonia* & 458 & 102 & Febrero & $\mathrm{R}$ \\
\hline Charadrius semipalmatus & 5864 & 1844 & Noviembre & M \\
\hline Charadrius vociferus* & 356 & 166 & Noviembre & $\mathrm{R}$ \\
\hline Haematopus palliatus $* \mathrm{P}$ & 77 & 18 & Marzo & $\mathrm{R}$ \\
\hline Himantopus mexicanus* & 31413 & 8079 & Noviembre & RI \\
\hline Recurvirostra americana & 138164 & 39947 & Enero & M \\
\hline Jacana spinosa $*$ & 290 & 58 & Febrero & $\mathrm{R}$ \\
\hline Actitis macularius & 307 & 62 & Diciembre & M \\
\hline Tringa melanoleuca & 838 & 242 & Noviembre & M \\
\hline Tringa semipalmata & 5217 & 1265 & Febrero & M \\
\hline Tringa flavipes & 1016 & 272 & Noviembre & M \\
\hline Numenius phaeopus & 642 & 163 & Febrero & M \\
\hline Numenius americanus & 1454 & 522 & Febrero & M \\
\hline Limosa fedoa & 13611 & 3189 & Marzo & M \\
\hline Arenaria interpres & 6 & 5 & Diciembre & M \\
\hline Arenaria melanocephala & 2 & 1 & Diciembre & M \\
\hline Calidris canutus $^{P}$ & 80 & 30 & Diciembre & M \\
\hline Calidris mauri & 147541 & 49344 & Diciembre & M \\
\hline Calidris minutilla & 9328 & 2460 & Diciembre & M \\
\hline Calidris alpina & 72 & 45 & Marzo & M \\
\hline Calidris himantopus & 5189 & 1670 & Diciembre & M \\
\hline Limnodromus spp. & 74102 & 24975 & Diciembre & M \\
\hline Gallinago delicata & 1 & 1 & Diciembre & M \\
\hline Phalaropus tricolor & 888 & 866 & Abril & M \\
\hline Phalaropus fulicarius & 4 & 4 & Diciembre & M \\
\hline Abundancia estimada & & 136236 & & \\
\hline
\end{tabular}

Se indican la abundancia acumulada y su abundancia (estimación) máxima. Presencia: $\mathrm{M}=$ Migratorio, $\mathrm{R}=\mathrm{Residente} \mathrm{y} \mathrm{RI}=$ Residentes con poblaciones migratorias. NOM-059: A= Amenazada y $\mathrm{P}=$ En peligro de extinción. * indica especies con reproducción en Marismas Nacionales. Total abundance and maximum count are presented. Presence: $\mathrm{M}=\mathrm{Migratory}, \mathrm{R}=$ Residents and $\mathrm{RI}=$ Residents species with migratory populations. NOM-059: $\mathrm{A}=$ Threatened, $\mathrm{P}=$ Endangered. * indicate breeding shorebirds.

(Charadrius nivosus Linnaeus 1758) se utilizó el estimado de Thomas et al. (2012).

\section{RESULTADOS}

Durante la temporada 2010-2011 se observaron 27 especies de aves playeras y un género (Limnodromus spp. Wied 1833), pertenecientes a cinco familias: Charadriidae (6 especies),
Haematopodidae (1), Recurvirostridae (2), Jacanidae (1) y Scolopacidae (18). Predominaron las especies migratorias (75\%), seguidas de las residentes (18\%) y de las especies que presentan tanto fracciones poblacionales residentes como migratorias (7 \%; Cuadro 1).

Entre las especies de aves playeras observadas en $\mathrm{MN}$, tres están incluidas en alguna categoría de protección definida por el 
Gobierno mexicano (DOF, 2010b). El Chorlo nevado (Charadrius nivosus Linnaeus 1758) se cataloga como amenazado y tanto el Playero rojizo del Pacífico (Calidris canutus roselaari Linnaeus 1758) como el Ostrero americano del Pacífico (Haematopus palliatus frazari Temminck 1820) se consideran en peligro de extinción (Cuadro I). Adicionalmente, en MN se registró la reproducción de seis especies de aves playeras (Cuadro I), siendo laguna Las Garzas-Chahuín la zona donde se registraron la mayoría de las especies anidantes como el Chorlo nevado, el Chorlo piquigrueso (Charadrius wilsonia Ord 1814), el Chorlo de doble collar (Charadrius vociferus Linnaeus 1758) y el Ostrero americano del Pacífico.

La riqueza específica presentó su valor más alto en diciembre con 26 especies. Entre enero y mayo esta mostró fluctuaciones con tendencia a la baja, hasta alcanzar el valor mínimo en verano (junio) con 17 (Fig. 2). La abundancia presentó su valor más alto en diciembre con 127000 aves, para posteriormente disminuir en enero, tendencia que se mantuvo hasta alcanzar su valor mínimo en junio con 2000 aves (Fig. 2). La suma de los máximos mensuales por especie indica que MN fue utilizada por al menos 136236 aves playeras (Tabla 1).

Espacialmente la riqueza fue mayor en las lagunas las Garzas-Chahuín con 26 especies y similar en Chumbeño y la Polca, con 21 especies. Laguna grande de Mexcaltitán fue donde se presentó la menor riqueza con sólo 6 especies (Fig. 3). El dendrograma de similitud mostró que las abundancias de las aves playeras se concentraron espacialmente en tres lagunas (Grupo 1, Fig. 3): Chumbeño (37\% del total), Las Garzas-Chahuin (24.2\%) y La Polca (24\%). En contraste, los sitios de menor importancia se registraron en la parte sur de MN (Grupo 3 y Mexcaltitan; menos del 3\% de la abundancia total), mientras que en Cañadas y en Laguna Zoyata (Grupo 2; parte central del sistema) las abundancias fueron del 6 y $7 \%$ del total, respectivamente. Temporalmente, las tendencias de las abundancias fueron similares en las lagunas del Grupo 1. En estas lagunas las aves arribaron entre octubre y noviembre y alcanzaron su máxima abundancia en diciembre.

Las especies más numerosas de $\mathrm{MN}$ fueron el Playerito occidental (Calidris Mauri Cabanis 1857, $33 \%$ de los registros totales), la Avoceta americana (Recurvirostra americana Gmelin 1789, $31 \%$ ) y los Costureros (Limnodromus spp., $17 \%$ ); estas especies acumularon más del $80 \%$ de la abundancia total. Temporalmente, el Playerito occidental arribó al área entre octubre y noviembre, con números de más de 30000 aves y alcanzó su máximo en diciembre (49

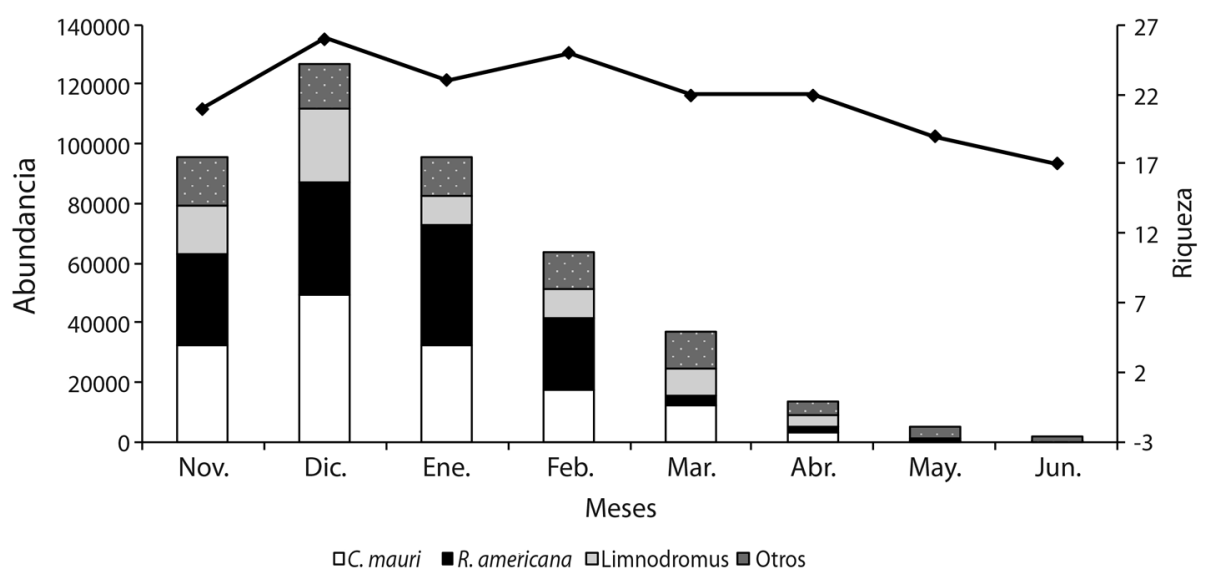

Fig. 2. Riqueza específica (linear) y abundancia (barras) temporal. La abundancia fue divida entre las tres especies más importantes en Marismas Nacionales y una categoría de otros donde se incluyen las especies restantes.

Fig. 2. Specific richness (line) and abundance per month (bars). Abundances were divided considering the three most important species at Marismas Nacionales and a category of others where remaining species are included. 

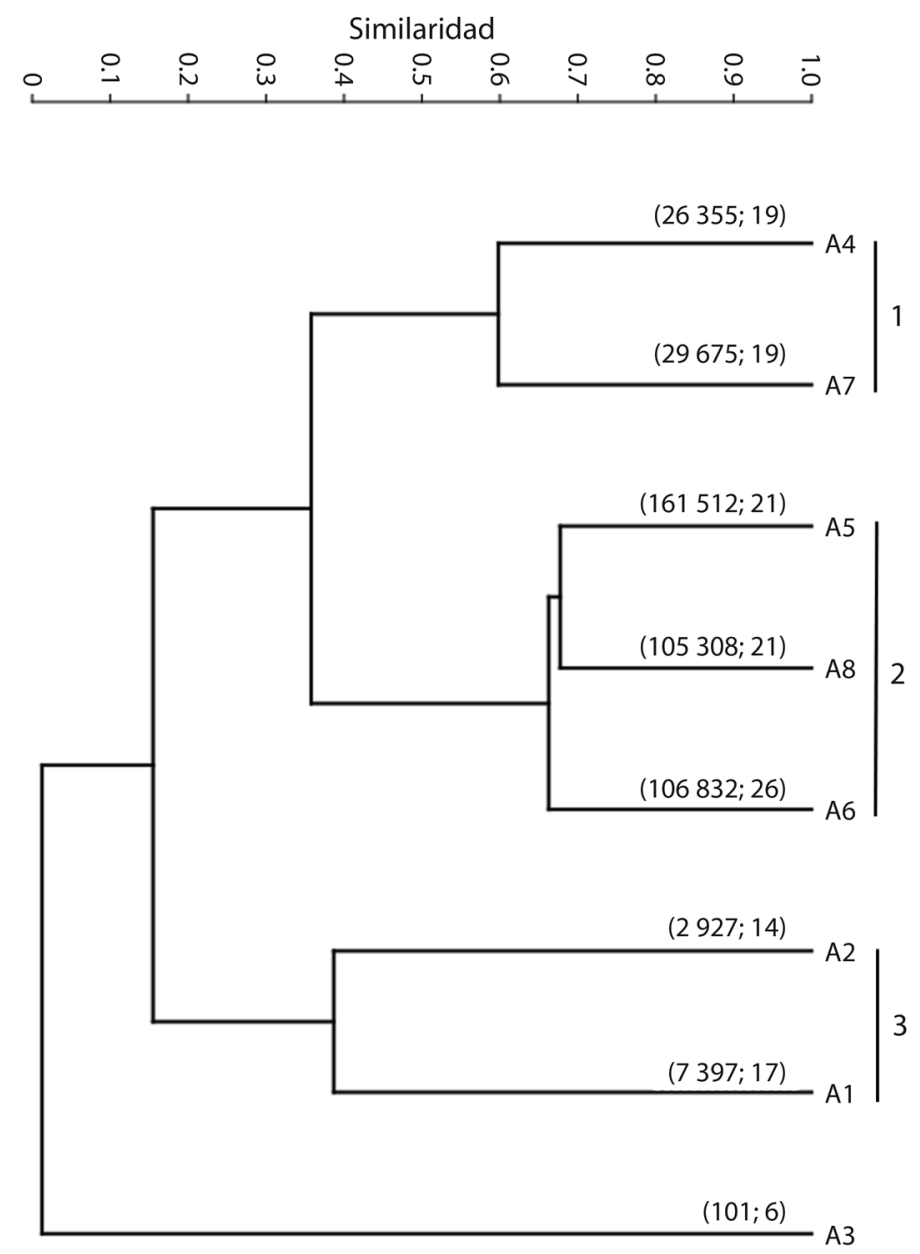

Fig. 3. Dendrograma de similitud utilizando el índice de Bray-Curtis para agrupar las áreas de Marismas Nacionales en función a la abundancia y riqueza. Entre paréntesis se señala la abundancia acumulada de las aves playeras y separada por un coma la riqueza especifica.

Fig. 3. Dendrogram of similarity using the Bray-Curtis index to group the Marismas Nacionales' areas according to abundance and richness. Parentheses indicate the accumulated abundance of shorebirds and separated by a comma the specific richness.

344). La abundancia de esta especie disminuyó gradualmente de enero hasta junio, cuando estuvo ausente del área de estudio (Fig. 2). La abundancia máxima observada en diciembre representó el $1.4 \%$ de su estimado poblacional.

La Avoceta americana, también en noviembre presentó números altos mismos que se incrementaron paulatinamente hasta alcanzar la mayor abundancia en enero (39947 aves). Posteriormente, dicha abundancia disminuyó entre febrero y marzo, para presentar sus números más bajos en junio (121 aves; Fig. 2). La abundancia máxima de la especie representó cerca del 9\% de su estimado poblacional. Los Costureros presentaron números altos en noviembre y alcanzaron sus máximos en diciembre (24 975 aves), posteriormente entre enero y marzo la abundancia se estabilizó con un promedio de 9500 aves, para disminuir en abril y estar ausentes en verano (Fig. 2). Finalmente, y aunque numéricamente no fue de las especies más importantes, la abundancia máxima registrada 
para el Chorlo nevado (268 aves) representan poco más del $6 \%$ del estimado para la población del Pacífico.

Las tres especies numéricamente más importantes se congregaron porcentualmente de manera diferente entre las localidades más relevantes de MN. En laguna Chumbeño se registraron porcentajes similares de Playerito occidental y de Avoceta americana (41 y $49.7 \%$ respectivamente), con baja importancia relativa de los Costureros. En laguna Las Garzas-Chahuín, la especie predominante fue el Playerito occidental (60\% de las abundancias de aves playeras) mientras que en laguna La Polca lo fue la Avoceta americana (49.4\%). Los Costureros presentaron porcentajes muy similares tanto en laguna Las Garzas-Chahuín como en laguna La Polca (25.3 y $23.3 \%$ respectivamente). La prueba estadística mostró que existe dependencia entre la abundancia de las especies con respecto a los sitios y mes de observación $\left(X^{2}{ }_{161}=140464, \mathrm{p}<0.01\right)$.

\section{DISCUSIÓN}

La riqueza específica de aves playeras observada en MN correspondió con lo esperado según la literatura (Escalante, 1988; Howell, \& Webb, 1995; Morrison \& Ross, 2009), excepto por el Falaropo pico grueso (Phalaropus fulicarius Linnaeus 1758), mismo que representa el primer registro para el área de estudio y que se observó en primavera y en baja abundancia (4 aves). La distribución invernal indicada para esta especie incluye las costas de $\mathrm{MN}$ y aunque es predominantemente pelágica, llega a observarse eventualmente en lagunas costeras (Stenzel, Hickey, Kjelmyr, \& Page, 2002; Ashoori, Barati, \& Reihannian, 2007; Carmona \& Danemann, 2013).

En MN se han reportado abundancias de aves playeras invernantes de entre $64000 \mathrm{y}$ 104000 aves (Harrington, 1993; Morrison et al., 1994; Ortega-Solis, 2011). Las abundancias registradas en este trabajo representan las más altas en los últimos años para el área de estudio, con 136236 aves. Estas diferencias están dadas principalmente por la metodología (censo aéreo vs censo terrestre) y el esfuerzo usado (el esfuerzo de este estudio comprendió ocho meses). La abundancia máxima observada se registró en diciembre, patrón semejante al observado en otros humedales del noroeste de México (Danemann, Carmona, \& Fernández, 2002; Carmona et al., 2011). Las abundancias menores correspondieron con la temporada reproductiva, época en la que al menos seis especies de aves playeras anidaron en $\mathrm{MN}$, por lo que se sugiere la necesidad de llevar a cabo estudios sobre la reproducción de estas especies, particularmente en lagunas Las Garzas-Chahuín.

La migración hacia el norte por parte de las aves playeras se caracteriza por presentar abundancias altas en los sitios de paso y recuperación (Recher, 1966; O’Reilly \& Wingfield, 1995; Carmona, Ruiz-Campos, \& Brabata, 2004), con los datos aquí generados no se refleja este incremento, lo que hace suponer que $\mathrm{MN}$ es poco utilizada durante esta época. Esta escasa utilización es consistente con otros humedales del noroeste de México, como en la península de Baja California (Carmona et al., 2004), el complejo lagunar de Guerrero Negro (Carmona et al., 2011) o Laguna San Ignacio (Mendoza \& Carmona, 2013). Por otro lado, y aunque este periodo no fue parte del estudio, durante las visitas prospectivas (septiembre y octubre) se observaron abundancias bajas de aves playeras, lo cual puede estar relacionado con la época de lluvia inmediata anterior, pues en $\mathrm{MN}$ la precipitación se concentra principalmente en junio y octubre (CONAGUA, 2004), por lo que la mayor parte de las planicies lodosas se encuentren inundadas y son muy poco utilizadas.

La zona que presentó la mayor riqueza fue laguna las Garzas-Chahuín, probablemente debido a su mayor heterogeneidad ambiental, ya que en ella existen amplias planicies lodosas de poca profundidad y un ambiente artificial (salinera en desuso), lo que permite a muchas especies alimentarse. Todas estas características ofrecen una amplia variedad de recursos espaciales y alimenticios (Myers et al., 1987; Yates et al., 1993; Danufsky \& Colwell, 2003). 
Las lagunas Chumbeño, Las GarzasChahuín y La Polca (donde se acumularon el $85 \%$ de los registros) son cuerpos de agua perenes que, al menos fuera de la época de lluvia, presentan profundidades que no superan los $20 \mathrm{~cm}$ en su periferia. Adicionalmente son las áreas con la mayor cobertura de planos lodosos (Carmona-Islas, Bello-Pineda, Carmona, \& Velarde, 2013). Las planicies lodosas intermareales son los ambientes que registran las mayores abundancias de aves playeras, debido a la alta disponibilidad de presas potenciales presentes en estos sustratos (Desholm, Harder, \& Mouritsen, 1998). Por ejemplo, en laguna Chumbeño se ha documentado una densidad alta de crustáceos e insectos, los cuales forman parte predominante en la dieta de muchas especies de aves playeras. Esta densidad de invertebrados se mantiene alta durante el periodo invernal, momento en el que se concentran las mayores abundancias de aves playeras (Molina, 2014). Por otro lado, en laguna Las Garzas-Chahuín un estudio técnico sobre invertebrados bentónicos registró una alta proporción de moluscos y anélidos (Carmona \& Bernal, 2015), mismos que también forman parte en la dieta de las aves playeras (Quammen, 1984).

Respecto a las tres especies más abundantes, el Playerito occidental ha sido indicado como el ave playera más numerosa en el noroeste de México. En MN se registraron cerca de 50000 individuos de esta especie, no obstante, esta zona no se encuentra entre los humedales de mayor relevancia en México para la especie, ya que en las costas del Estado de Sinaloa se han registrado sitios donde el Playerito occidental es más numerosa (e. g. Bahía Santa María y laguna Huizache-Caimanero son utilizadas por 300000 aves de esta especie; Engilis et al., 1998; Carmona \& Danemann, 2013; 2014). Las mayores abundancias del Playerito occidental en $\mathrm{MN}$ se presentaron entre noviembre y enero, lo que reafirma la idea de que el área de estudio es una zona de inverno para la especie. La abundancia máxima se registró en diciembre, lo que concuerda con lo observado en otros humedales del noroeste de
México como laguna San Ignacio (Mendoza \& Carmona, 2013) o el complejo lagunar Guerrero Negro (Carmona et al., 2011).

La Avoceta americana es una de las especies más abundantes en la parte continental del noroeste de México, desde el sur de Sonora hasta Nayarit (Morrison et al., 1994; Engilis et al., 1998; Molina, 2014). La abundancia aquí registrada (c.a. 40000 aves) ubica a $\mathrm{MN}$ como uno de los sitios más utilizados de la región (Engilis et al., 1998; Morrison \& Ross, 2009; Carmona \& Danemann, 2013). Los números máximos se presentaron en enero, lo que concuerda con otros humedales importantes de invernación para la especie, como la Bahía de Humboldt, California (Evans \& Harris, 1994). La abundancia de la Avoceta americana disminuyó notoriamente a partir de marzo, dada la partida de las aves hacia sus sitios de reproducción, ya que la anidación puede comenzar a partir de abril (Cavitt, 2006; Ackerman et al., 2013). La distribución espacial de la Avoceta americana se concentró en las tres principales áreas de $\mathrm{MN}$, estos sitios son lagunas perenes con profundidades bajas y con una influencia mínima de marea, características que favorecen la concentración de grandes bandadas de esta especie (Boettcher, Haig, \& Bridges, 1994; Ackerman et al., 2013).

Los Costureros están entre los taxa más frecuentes dentro del corredor migratorio del Pacífico y en general están bien representados numéricamente en las comunidades de aves playeras de los humedales del noroeste de México (Page et al., 1997; Engilis et al., 1998; Morrison \& Ross, 2009; Ortega-Solis, 2011). Durante la temporada no reproductiva se pueden registrar dos especies del género Limnodromus (L. griseus Gmelin 1789 y L. scolopaceus Say 1823) en MN, no obstante, durante esta época se dificulta diferenciarlos mediante características morfológicas, por lo que se recomienda agruparlos y reportar su información como género (Jehl, Klima, \& Harris, 2001; Morrison \& Ross, 2009). En MN la importancia numérica de este género ha variado entre 8000 y 10000 (Morrison et al., 1994; OrtegaSolis, 2011). Las abundancias en el presente 
estudio (25000 aves) refleja una alta variabilidad interanual, más aún al considerar que MN es un humedal tropical con alto dinamismo en su régimen hidrológico (Blanco et al., 2011). Estas diferencias también pueden deberse a la metodología utilizada pues Morrison et al. (1994) utilizó un censo aéreo mientras que en el presente estudio fue un recorrido terrestre donde existe una mayor facilidad de detección en los grupos de aves. Los Costureros prefieren humedales lodosos para su alimentación, lo que caracterizó sus principales zonas de distribución en MN (Las Garzas, Chumbeño y La Polca; Engilis et al., 1998).

En suma, el estudio presente determinó la abundancia general de aves playeras que utilizan $\mathrm{MN}$, así como los sitios que concentran los mayores números de este grupo de aves; estos sitios deben ser prioridad en los planes de conservación. Los datos obtenidos en el trabajo presente pueden permitir la elaboración de programas de monitoreo los cuales se fundamentan en un sólido conocimiento de la distribución de las especies dentro de un humedal. Finalmente, las abundancias de aves playeras aquí reportadas actualizan la información sobre este sitio y permiten mantener su categoría ante la Red Hemisférica de Reservas para las Aves Playeras.

Declaración de ética: los autores declaran que todos están de acuerdo con esta publicación y que han hecho aportes que justifican su autoría; que no hay conflicto de interés de ningún tipo; y que han cumplido con todos los requisitos y procedimientos éticos y legales pertinentes. Todas las fuentes de financiamiento se detallan plena y claramente en la sección de agradecimientos. El respectivo documento legal firmado se encuentra en los archivos de la revista.

\section{AGRADECIMIENTOS}

A Pronatura Noroeste oficina Tepic particularmente a Mauricio Cortés y César Rodríguez por su apoyo en el trabajo de campo. Este trabajo fue parcialmente apoyado por el
Proyecto "Planificación de mecanismo para la conservación legal y restauración de las poblaciones de aves acuáticas migratorias invernantes en Marismas Nacionales, Nayarit, México", auspiciado por North American Wetland Conservation Act, agradecemos particularmente el apoyo de Ellen Murphy. También se recibió apoyo de Kennecott Utah Copper Corporation, and the Rio Tinto-Bird Life Program, por lo que se agradece a Jonathan Stacey e Ítala Yepes, al equipo del Secretariado de las Américas de BirdLife International y a los miembros de Linking Communities. Al personal de la Reserva de la Biosfera Marismas Nacionales Nayarit, en particular a Víctor Hugo Vázquez Morán (director) y Daniela Valera Aguilar (programa de monitoreo). A Yuri Albores-Barajas por sus comentarios al manuscrito. GCA, PML y JCA agradecen los apoyos otorgados por COFAA y EDI del Instituto Politécnico Nacional.

\section{RESUMEN}

El noroeste de México alberga las mayores abundancias de aves playeras del país. Sin embargo, para muchos de estos humedales, información sobre comunidades de aves playeras, como abundancia, tendencias poblacionales y riqueza es limitada. Actualmente, los sitios de descanso son críticos para la conservación porque las poblaciones de aves playeras han decaído en las últimas décadas. Marismas Nacionales (MN) es un humedal tropical importante con un ecosistema dinámico donde los estudios de aves playeras están limitados a unos pocos censos aéreos y terrestres. Así el objetivo del trabajo fue describir la abundancia y distribución espacial y temporal de las aves playeras en MN (temporada 2010-2011). Se seleccionaron ocho unidades de muestreo en las que se llevaron a cabo censos mensuales (noviembre 2010 a junio 2011). Se determinaron las riquezas y abundancias por sitio-mes, además se realizó un análisis espacial y temporal de las especies dominantes. Se registraron 27 especies de aves playeras y un género, con un estimado mínimo de 136236 individuos. Este número hace a $\mathrm{MN}$ uno de los humedales prioritarios para la conservación en México, pues alberga al $10 \%$ de la abundancia general del noroeste. Las especies dominantes fueron el Playerito occidental (Calidris mauri, $33 \%$ del total), la Avoceta americana (Recurvirostra americana, $31 \%$ ) y los Costureros (Limnodromus spp., $17 \%$ ). Espacialmente las lagunas de mayor importancia fueron: Chumbeño (37\% del total registrado), Las Garzas-Chahuin (24\%) y La Polca (24\%). Este trabajo actualiza la información sobre aves playeras que utilizan $\mathrm{MN}$ y podría permitir el establecimiento de un programa de monitoreo, lo 
cual es prioritario sobre todo porque el área es un Sitio de Importancia Internacional por parte de la Red Hemisférica de Reserva para las Aves Playeras.

Palabras claves: laguna tropical; aves playeras; temporada no reproductiva; comunidad de aves.

\section{REFERENCIAS}

Ackerman, J., Hartman, A., Herzog, M., Robinson, J. A., Oring, L. W., Skorupa, J. P.,... Boettcher, R. (2013). American Avocet (Recurvirostra americana). The Birds of North America Online (A. Poole, Ed.). Issue 275. Ithaca: Cornell Lab of Ornithology. Recuperado de https://doi.org/10.2173/bna.275

Andres, B., Smith, P., Morrison, R. I. G., Gratto-Trevor, C., Brown, S., \& Friis, C. (2012). Population estimates of North American shorebirds, 2012. Wader Study Group Bulletin, 119(3), 178-194.

Arce, N., Carmona, R., Miramontes, E., Ayala-Pérez, V., Hernández-Alvarez, A., \& Mendoza, L. F. (2015). An overwintering group of Red Knots (Calidris canutus roselaari) in Las Garzas lagoon, Nayarit, México. Water Study, 122(2), 135-141.

Ashoori, A., Barati, A., \& Reihannian, H. R. (2007). Recent observations of the Red Phalarope phalaropes fulicarius at Boujagh National Park, Gilan province, and And Agh Gol wetland, Hamedan province and its status in Iran. Podoces, 2(2), 141-162.

Berlanga-Robles, C., García-Campos, R. R., López-Blanco, J., \& Ruiz-Luna, A. (2010). Patrones de cambio de coberturas y usos del suelo en la región costa norte de Nayarit (1973-2000). Investigaciones Geográficas, Boletín del Instituto de Geografia, UNAM, 72(1), 7-22.

Blanco, M., Flores-Verdugo, F., Ortiz-Pérez, M., de la Lanza, G., López-Portillo, J.,... González, A. (2011). Diagnóstico funcional de Marismas Nacionales. Informe final. Nayarit, México: Convenios de Coordinación. Universidad Autónoma de Nayarit y Comisión Nacional Forestal.

Boettcher, R., Haig, S., \& Bridges Jr., W. (1994). Behavioral patterns and nearest neighbor distances among non-breeding American avocets. The Condor, 96(4), 973-986.

Bojórquez-Tapia, L. A., Díaz-Mondragón, S., \& Ezcurra E. (2001). GIS-based approach for participatory decision making and land suitability assessment. International Journal of Geographical Information Science, 15(2), 129-151.

Carmona, R., Ruiz-Campos, G., \& Brabata, G. (2004). Seasonal abundance of migrant shorebirds in Baja California Peninsula, Mexico, and California, USA. Wader Study Goup Bulletin, 105(1), 65-70.
Carmona, R., Arce, N., Ayala-Pérez, V., \& Danemann, G. D. (2011). Seasonal abundance of shorebirds at the Guerrero Negro wetland complex, Baja California, Mexico. Wader Study Group Bulletin, 118(1), 40-48.

Carmona, R., \& Danemann, G. D. (2013). Monitoreo de aves playeras migratorias en 11 sitios prioritarios del Noroeste de México y reconocimiento invernal de cinco sitios adicionales. México: Comisión Nacional de Áreas Naturales Protegidas.

Carmona, R., \& Danemann, G. D. (2014). Monitoreo de aves playeras migratorias en 11 sitios prioritarios del Noroeste de México y reconocimiento de cuatro sitios adicionales. México: Comisión Nacional de Áreas Naturales Protegidas.

Carmona, R., \& Bernal, T. (2015). Distribución espaciotemporal de aves playeras y su relación con los invertebrados bentónicos en la Reserva de la Biosfera Marismas Nacionales Nayarit, México (Informe Final). D.F. México: CONABIO.

Carmona-Islas, C., Bello-Pineda, J., Carmona, R., \& Velarde, E. (2013). Modelo espacial para la detección de sitios potenciales para la alimentación de aves playeras migratorias en el noroeste de México. Huitzil, 14(1), 22-42.

Cavitt, J. (2006). Great Salt Lake Shorebird Productivity: Bear River Migratory Bird Refuge, Farmington Bay Waterfowl Management Area, Great Salt Lake Shorelands Preserve. USA: Weber State University.

Cervantes-Abrego, M. (2000). Marismas Nacionales. En M. del Coro-Arizmendi \& L. Márquez-Valdelamar (Eds.), Áreas de Importancia para la Conservación de las Aves en México. México: CONABIO.

Comisión Nacional del Agua (CONAGUA). (2004). Base de datos del Clima en Nayarit. Gerencia Estatal en Nayarit. Departamento de Meteorología. México: CONAGUA.

Comisión Nacional de Áreas Naturales Protegidas (CONANP). (2005). Estudio previo justificativo para el establecimiento del área natural protegida: Área de Protección de Flora y Fauna Marismas Nacionales, Nayarit. México: SEMARNAT.

Crisci, J. V., \& López, M. F. (1983). Introducción a la teoría y práctica de la taxonomía numérica. Washington, USA: Organización de Estados Americanos.

Danufsky, T., \& Colwell, M. (2003). Winter shorebird communities and tidal flat characteristics at Humboldt Bay, California. The Condor, 105(1), 117-129.

Danemann, G., Carmona, R., \& Fernández, G. (2002). Migratory shorebirds in the Guerrero Negro saltworks, Baja California Sur, Mexico. Wader Study Group Bulletin, 97(1), 36-41. 
Desholm, M. D., Harder, A. M., \& Mouritsen, K. N. (1998). The sediment-plane: an alternative tool for estimating prey accessibility to tactilely feeding waders. Wader Study Group Bulletin, 85(1), 73-75.

Diario Oficial de la Federación (DOF). (2010a). Decreto por el que se declara como área natural protegida, con el carácter de reserva de la biosfera, la región conocida como Marismas Nacionales Nayarit. D.F., México: SEMARNAT

Diario Oficial de la Federación (DOF). (2010b). Norma Oficial Mexicana NOM-059-ECOL-2010, Protección ambiental-especies nativas de México de flora $y$ fauna silvestre categorías de riesgos y especificaciones para su inclusión, exclusión o cambio-lista de especies en riesgo. D. F., México: SEMARNAT.

Engilis, A., Oring, L. W., Carrera, E., Nelson, J. W., \& Martínez-López, A. (1998). Shorebird surveys in Ensenada Pabellones and Bahía Santa María, Sinaloa, México; Critical winter habitats for Pacific flyway shorebirds. Wilson Bulletin, 110(3), 332-341.

Escalante, P. (1988). Aves de Nayarit. México: Coordinación General de Enseñanza Superior Universidad Autónoma de Nayarit.

Evans, T. J., \& Harris, S. W. (1994). Status and habitat use by American Avocets wintering at Humboldt Bay, California. The Condor, 96(1), 178-189.

Harrington, B. A. (1993). A coastal aerial winter shorebird survey on the Sonora and Sinaloa coasts of Mexico, January 1992. Wader Study Group Bulletin, 67(1), 44-49.

Hayman, P., Marchant, J., \& Prater, T. (1988). Shorebirds: an identification guide. New York, USA: Houghton Mifflin.

Howell, S. N. G., \& Webb, S. (1995). A guide to the birds of México and Northern Central America. New York, USA: Oxford University Press.

Howes, J., \& Bakewell, D. (1989). Shorebirds Studies Manual. Kuala Lumpur, Malasia: Asian Wetland Bureau.

Jehl, Jr., J. R., Klima, J., \& Harris, R. E. (2001). Shortbilled Dowitcher (Limnodromus griseus). Birds of North America Online (A. Poole, Ed.). Ithaca: Cornell Lab of Ornithology. Recuperado de https://doi. org/10.2173/bna.564

Mendoza, L. F., \& Carmona, R. (2013). Distribución espacial y temporal de aves playeras (Orden: Charadriiformes) en Laguna San Ignacio, Baja California Sur, México. Revista de Biología Tropical 61(1), 229-241.

Molina, D. (2014). Patrones de uso invernal de avoceta americana (Recurvirostra americana, Gmelin 1789) en Marismas Nacionales, Nayarit, México (Tesis de
Maestría). Universidad Autónoma de Baja California Sur, Baja California Sur, México.

Morrison, R. I. G., Ross, R. K., \& Guzmán, J. (1994). Preliminary results of surveys of the southern half of the Pacific coast, states of Chiapas to Sinaloa. Ottawa, Canada: Canadian Wildlife Service.

Morrison, R. I. G., \& Ross, R. K. (2009). Atlas of Nearctic shorebirds on the coast of Mexico. Ottawa, Canada: Canadian Wildlife Service.

Myers, J. P., Morrison, R. I. G., Antas, P. Z., Harrington, B. A., Lovejoy, T. E., Sallaberry, M.,... Tarak, A. (1987). Conservation strategy for migratory species. American Scientist, 75(1), 19-26.

O'Brien, M., Crossley, R., \& Karlson, K. (2006). The Shorebird Guide. New York, USA: Houghton Mifflin.

O’Reilly, K. M., \& Wingfield, J. C. (1995). Spring and autumn migration in arctic shorebirds: some distance, different strategies. American Zoologist, 35(3), 222-233.

Ortega-Solis, L. (2011). Distribución especial y temporal de las aves playeras invernantes en la Reserva de la Biosfera Marismas Nacionales, Nayarit (Tesis de Licenciatura). Universidad Autónoma de Nayarit, Nayarit, México.

Page, G., Palacios, E., Alfaro, L., González, S., Stenzel, L., \& Jungers, M. (1997). Numbers of wintering shorebirds in coastal wetlands of Baja California, México. Journal of Field Ornithology, 68(4), 562-574.

Quammen, M. L. (1984). Predation by shorebirds, fish, and crabs on invertebrates in intertidal mudflats: an experimental test. Ecology, 65(2), 529-537.

Recher, H. F. (1966). Some aspects of the ecology of migrant shorebirds. Ecology, 47(3), 393-407.

Skagen, S., \& Knopf, F. (1994). Migrating shorebirds and habitat dynamics at a prairie wetland complex. Wilson Bulletin, 106(1), 91-105.

Stenzel, L., Hickey, C., Kjelmyr, J., \& Page, G. (2002). Abundance and distribution of shorebirds in the San Francisco Bay area. Western Birds, 33(2), 69-98.

Thomas, S. M., Lyons, J. E., Andres, B. A., Elliot-Smith, E., Palacios, E., Cavitt, J. F.,... Zimmerman, T. (2012). Population size of Snowy plover breeding in North America. Waterbirds, 35(1), 1-14.

Yates, M. G., Goss-Custard, J. D., McGrorty, S., Lakhani, K. H., Dit Durell, S., Clarke, R. T.,... Frost, A. J. (1993). Sediment characteristics, invertebrate densities and shorebird densities on the inner banks of the Wash. Journal of Applied Ecology, 30(4), 599-614.

Zar, J. (2010). Biostatistical Analysis. New Jersey, USA: Pearson Prentice Hall. 